\title{
GSTA1*-69C/T and GSTO2*N142D as asthma- and allergy-related risk factors in Italian adult patients
}

\author{
Sara Piacentini, $*$ Renato Polimanti,* Andrea Iorio, ${ }^{\dagger}$ Maurizio Cortesi, ${ }^{*}$ Fabrizio Papa, \\ Mauro Rongioletti, ${ }^{*}$ Giancarlo M. Liumbruno, ${ }^{*}$ Dario Manfellotto ${ }^{\dagger}$ and Maria Fuciarelli* \\ *Department of Biology, University of Rome 'Tor Vergata', ${ }^{\dagger}$ Clinical Pathophysiology Centre and ${ }^{\ddagger}$ Clinical Pathology \\ Department, Fatebenefratelli Association for Biomedical Research (AFaR)-'San Giovanni Calibita' Fatebenefratelli
}

Hospital, Rome, Italy

\section{SUMMARY}

1. Asthma and allergies are characterized by variable and subjective symptoms influenced by many genes, molecular mechanisms and environmental factors. The presence of inflammation and oxidative stress in the airways are important biochemical features of asthma and respiratory allergies. Glutathione Stransferase (GSTs) enzymes play an important role in cellular protection against inflammation, and functional genetic polymorphisms in GST genes show a significant association with asthma and allergy risk. Specifically, our previous study on asthmatic children highlighted GSTA1 and GSTO2 as novel susceptibility loci for asthma.

2. In the present study we focused our attention on GSTA1*-69C/T (rs3957357) and GSTO2*N142D (rs156697) polymorphisms to confirm our previous results in an independent adult study population and to clarify whether GSTA1 and GSTO2 gene polymorphisms are involved in a non-discriminative pathway towards asthma and respiratory allergy.

3. To accomplish this, we recruited $\mathbf{1 0 3}$ patients with respiratory allergies, 199 patients with asthma and 200 healthy controls. Genomic DNA extracted from buccal cells was screened for GSTA1*-69C/T and GSTO2*N142D single nucleotide polymorphisms.

4. The GSTA1*-69T and GSTO2*D142 variants are both associated with a significantly increased risk of asthma, whereas only GSTA1*-69C/T is significantly associated with allergies. These outcomes confirm the involvement of GSTO2 loci in asthma and suggest that GSTA1 is a common risk factor for asthma and allergies.

Key words: genetic factors, glutathione S-transferases, GSTA1, GSTO2, respiratory diseases, single nucleotide polymorphisms.

Correspondence: Maria Fuciarelli, Department of Biology, University of Rome 'Tor Vergata', Via della Ricerca Scientifica, 100133 Rome, Italy. Email: fuciarelli@uniroma2.it

Received 7 November 2013; revision 16 January 2014; accepted 18 January 2014.

(C) 2014 Wiley Publishing Asia Pty Ltd

\section{INTRODUCTION}

Asthma is a common chronic inflammation of the respiratory tracts; the cause and the nature of its pathogenesis have not been well established as yet. However, it is known that asthma is a complex multigenic disorder determined by the complicated interaction of genetics with the environment. ${ }^{1}$ To date, there is not a clear definition of the asthma phenotype and, for this reason, researchers have focused their attention on several characteristics that can be measured objectively, such as atopy, airway hyperresponsiveness and allergen sensitization. Rackeman first reported the distinction of two clinical patterns of asthma: a form in which extrinsic factors of the disease were triggering the symptoms (allergic asthma) and another in which no peculiar triggering factor could be found (non-allergic asthma). ${ }^{2}$ Ever since the first description of non-allergic asthma, there has been debate about the relationship of the disease to atopy. ${ }^{3}$ Non-allergic asthma could represent a form of autoimmunity, or auto-allergy, triggered by infection because a respiratory influenza-like illness often precedes onset. ${ }^{4}$ The existence of two distinct asthma phenotypes suggests that their respective genetic predispositions could be different. However, although several genes have been shown to be linked to asthma, no relationship to the allergy was reported. ${ }^{5}$ Although a host allergy is a recognized risk factor for airway inflammation, atopy alone cannot cause asthma. Indeed, results of different epidemiological studies have consistently shown that allergies and asthma often coexist in the same patient. Moreover, allergies are a risk factor for asthma and, despite the severe impact on patients and society as a whole, respiratory allergies are neglected and/or under-recognized. ${ }^{6}$ Therefore, some genes may be common for asthma and allergies and some genes may be disease-specific markers.

The discovery of specific markers for asthmatic and allergic phenotypes would help greatly in recognizing the different entities of these respiratory syndromes. At the clinical level, the discovery of these markers would also help facilitate the prevention and treatment of the diseases in individuals. ${ }^{7}$ The presence of inflammation in the airways is an important common feature of asthma and respiratory allergies. In particular, attention is mainly focused on oxidative stress, a key component of inflammation. ${ }^{8}$ In recent years a number of genome-wide association studies (GWAS) in asthma or allergy phenotypes have described novel and interesting gene polymorphisms, but have also set the role of some anti-oxidant genes previously described as being involved 
in this scenario. ${ }^{9}$ However, the genetic component involved in respiratory disease development or symptom worsening has not been completely clarified. ${ }^{7,10}$ Indeed, the GWAS approach may underestimate the genomic and environmental contexts, leaving uncovered loci involved in gene-environment interaction, such as detoxification genes. ${ }^{11}$

Several studies have demonstrated that some changes in the function of the xenobiotics detoxification system result in an increased susceptibility to asthma development or symptom worsening. ${ }^{8}$ Recently, interest has focused on genes encoding the glutathione S-transferases (GSTs), the principal Phase II enzyme superfamily. ${ }^{12}$ For GST enzymes, functional polymorphisms have been shown to be risk factors for common diseases and for xenobiotics-related susceptibility. ${ }^{13-16}$ Regarding GST genes and respiratory phenotypes, most of the studies have analysed the association of GSTM1, GSTP1 and GSTT1 gene polymorphisms to disease risk, with contrasting results. ${ }^{17-19}$ Recent meta-analyses have highlighted that the genetic associations of GSTM1, GSTP1 and GSTT1 to asthma are not statistically significant. ${ }^{20,21}$ In addition, high heterogeneity is present among the studies. ${ }^{20,21}$ This outcome is probably due not only to publication bias and sample size, but also to the extreme gene-environment interactions present in the asthmatic phenotypes and to the genetic differences present among human populations. ${ }^{20,22}$ Our previous case-control study recently associated two functional polymorphisms of GSTA1 and GSTO2 enzymes (GSTA1*-69C/T and GSTO2*N142D) with asthma susceptibility in Italian children. ${ }^{23}$ Although no other genetic association studies have investigated these variants and none of the GWAS have found these polymorphisms to be significant, some evidence seems to confirm our association hypothesis. In 2007, the Framingham Heart genomewide association study hypothesized a role for GSTO2 as a credible candidate for a gene associated with pulmonary function. ${ }^{24}$ Sohn et al. highlighted that GSTA1 is upregulated in asthmatic airways, suggesting that it plays an important role in protecting the airways from oxidative stress. ${ }^{25}$ In order to confirm the role of GSTA1 and GSTO2 as susceptibility loci for asthma and to understand their involvement in allergies, we investigated GSTA1*-69C/T and GSTO2*N142D polymorphisms in three groups: asthmatics, allergy patients and healthy controls.

\section{METHODS}

\section{Study population}

The participants were recruited from the 'San Giovanni Calibita' Fatebenefratelli Hospital in Rome, Italy. The sample consisted of 103 patients with respiratory allergies (i.e. rhinitis, conjunctivitis, sinusitis), 199 patients with asthma (148 atopic and 51 non-atopic) and 200 healthy controls living in the same geographical area. The allergy patients were recruited from the Clinical Pathology Department, whereas both the asthmatic and healthy controls were recruited from the Clinical Pathophysiology Centre. Respiratory allergy diagnosis was based on a current physician's diagnosis, positivity to Prick and Rast tests and the use of antiallergic medication. To exclude an asthmatic component in allergic patients, we selected allergic patients without bronchial hyperresponsiveness. As reported previously, ${ }^{35}$ asthma was diagnosed in accordance with Global Initiative on Asthma criteria: ${ }^{29}$ (i) a current physician diagnosis of asthma; (ii) symptoms; and (iii) the use of anti-asthma medication. Ethnicity-matched controls were selected from non-asthmatic, non-atopic, healthy individuals with normal lung function who were visiting Fatebenefratelli Hospital for an annual check-up.

\section{Genotyping procedures}

Buccal cells were collected with an oral swab from each participant. Written informed consent was obtained from all individuals and the study was approved by the hospital's ethics committee. DNA from buccal cells was obtained using the phenol : chloroform : isoamylic alcohol method. ${ }^{36}$ The GSTA1*-69C/T and GSTO2*N142D polymorphisms were detected as reported previously. ${ }^{22}$ To ensure the reliability of the results, approximately $15 \%$ of the samples were randomly selected and analysed independently by a second researcher using the same protocol; in all cases the outcome was concordant.

\section{Statistical analyses}

The $\chi^{2}$-test and Student's $t$-test were used for the analysis of categorical and quantitative variables, as appropriate. The results of the genotyping procedures were used to verify Hardy-Weinberg equilibrium. Because we investigated two genetic markers in control and patient groups, the standard significance level for genetic association analysis has been adjusted to $\alpha=0.05 / 2=0.025$, according to the application of Bonferroni correction for multiple testing. Power analysis was performed using the Power for Genetic Association version 2.0. ${ }^{37}$ The minimum detectable effect with odd ratios (ORs) was calculated in a codominant genetic model, based on an $\alpha$ of 0.025 , an asthma prevalence of $6 \%$ and an allergy prevalence of $30 \%$ in the general population. With this sample size, we had $80 \%$ power to detect an OR of 1.57 for asthma association and an OR of 1.77 for allergy association if the risk allele frequency was higher than $25 \%$. The ORs and $95 \%$ Confidence Intervals (CIs) were calculated to evaluate the association between GST variants and asthma and GST variants and respiratory allergic disease. To estimate the ORs, different genetic models were considered: codominant (each genotype has a different disease risk), dominant (one copy of the allele is sufficient to increase the disease risk), recessive (two copies of the allele are necessary to increase the disease risk) and log-additive ( $r$-fold increased risk for one copy of the allele, $r^{2}$ increased risk for two copies of the allele). To evaluate the best genetic model, the Akaike information criterion (AIC) and Bayesian information criterion (BIC) were used.

\section{RESULTS}

Demographic and clinical characteristics of the groups investigated are given in Table 1. No significant differences were found between asthmatic patients and healthy controls in terms of age distribution, sex ratio or any of the other characteristics analysed. Conversely, significant differences were found for age and body mass index (BMI) distribution among allergy patients and healthy controls $(P<0.001)$. Data regarding the allelic and the genotype frequencies are reported in Table 2. All genotype frequencies in our controls were within the ranges reported previously in Italy; ${ }^{26}$ 
Table 1 Characteristics of asthmatic and allergic patients and healthy controls

\begin{tabular}{lcccc}
\hline & Asthmatic & Allergic & Control & $P$-value \\
\hline$n$ & 199 & 103 & 200 & \\
Age (years) & $52.4 \pm 1.2$ & $40.0 \pm 1.5$ & $54.9 \pm 1.0$ & $P_{\mathrm{a}}=0.110$ \\
& & & & $P_{\mathrm{b}}<0.001$ \\
Gender & & & & \\
$\quad \%$ Men & $82(41)$ & $42(41)$ & $89(44)$ & $P_{\mathrm{a}}=0.570$ \\
$\quad \%$ Women & $117(59)$ & $61(59)$ & $111(56)$ & $P_{\mathrm{b}}=0.384$ \\
BMI (kg/m $\left.{ }^{2}\right)$ & $25.3 \pm 0.3$ & $23.6 \pm 0.4$ & $25.0 \pm 0.2$ & $P_{\mathrm{a}}=0.405$ \\
& & & & $P_{\mathrm{b}}<0.001$ \\
Atopy & & & & \\
$\quad$ Atopic (\%) & $148(74)$ & $103(100)$ & $0(0)$ & - \\
$\quad$ Non-atopic (\%) & $51(26)$ & $0(0)$ & $200(100)$ & \\
Smoking habits & & & & \\
$\quad$ Smoker (\%) & $41(21)$ & $20(20)$ & $41(21)$ & $P_{\mathrm{a}}=0.036$ \\
$\quad$ Ex-smoker (\%) & $42(21)$ & $25(24)$ & $64(32)$ & $P_{\mathrm{b}}=0.291$ \\
$\quad$ Non-smoker (\%) & $116(58)$ & $58(56)$ & $95(47)$ & \\
Passive & $78(39)$ & $46(45)$ & $61(31)$ & $P_{\mathrm{a}}=0.085$ \\
smoking (\%) & & & & $P_{\mathrm{b}}=0.015$ \\
& & & & \\
Occupational & $62(33)$ & $21(20)$ & $48(24)$ & $P_{\mathrm{a}}=0.137$ \\
$\quad$ risk (\%) & & & & $P_{\mathrm{b}}=0.478$ \\
& & & & \\
\hline
\end{tabular}

Data show the mean \pm SD or the number of subject with percentages in parentheses, as appropriate.

$P$-values were calculated comparing asthmatics with controls $\left(P_{\mathrm{a}}\right)$ and allergic patients with controls $\left(P_{\mathrm{b}}\right)$. Significance was set at $P<0.05$.

Table 2 Allele and genotype distribution of GSTA1 and GSTO2 variants in asthmatics, allergic patients and healthy controls

\begin{tabular}{lcccc}
\hline & Asthmatic & Allergic & Control & $P$-value \\
\hline GSTA1*69C/T (rs3957357) & & & \\
-69C (\%) & $233(59)$ & $121(59)$ & $300(75)$ & $P_{\mathrm{a}}<0.001$ \\
-69T (\%) & $165(41)$ & $85(41)$ & $100(25)$ & $P_{\mathrm{b}}<0.001$ \\
-69C/-69C (\%) & $60(30)$ & $33(32)$ & $113(57)$ & $P_{\mathrm{a}}<0.001$ \\
-69C/-69T (\%) & $113(57)$ & $55(53)$ & $74(37)$ & $P_{\mathrm{b}}<0.001$ \\
-69T/-69T (\%) & $26(13)$ & $15(15)$ & $13(6)$ & \\
GSTO2*N142D (rs156697) & & & \\
N142 (\%) & $242(61)$ & $142(69)$ & $288(72)$ & $P_{\mathrm{a}}<0.001$ \\
D142 (\%) & $156(39)$ & $64(31)$ & $112(28)$ & $P_{\mathrm{b}}=0.431$ \\
N142/N142 (\%) & $77(39)$ & $48(47)$ & $98(49)$ & $P_{\mathrm{a}}<0.001$ \\
N142/D142 (\%) & $88(44)$ & $46(45)$ & $92(46)$ & $P_{\mathrm{b}}=0.444$ \\
D142/D142(\%) & $34(17)$ & $9(8)$ & $10(5)$ & \\
\hline
\end{tabular}

Data show the number of subject with percentages in parentheses.

$P$-values $\left(\chi^{2}\right)$ are reported.

$P$-values were calculated comparing asthmatics with controls $\left(P_{\mathrm{a}}\right)$ and allergic patients with controls $\left(P_{\mathrm{b}}\right)$.

Significance was set at $P=0.025$ (two-tailed after Bonferroni's adjustment).

the genotype frequencies were in Hardy-Weinberg equilibrium in the patient and control cohorts. The ORs and 95\% CIs calculated for different genetic models are given in Table 3. Regarding asthmatic versus control comparisons, both the polymorphisms studied showed an association with asthma risk. Regarding GSTA1, the OR analysis highlighted significant results for the codominant model (CT genotype: OR 2.74, 95\% CI 1.72-4.35; TT genotype OR 3.08, 95\% CI 1.41-6.72; $P<0.001$ ), dominant model (OR 2.79; 95\% CI 1.79-4.35; $P<0.001$ ) and log-additive model (OR $2.10 ; 95 \%$ CI $1.48-2.97 ; P<0.001)$. To detect the best model for our data, we used two standard model selection criteria: the AIC and BIC. The dominant model achieved the best scores for AIC and BIC. Regarding GSTO2, the OR analysis revealed significant outcomes for the codominant model (ND genotype: OR 1.20 , 95\% CI $0.76-1.89$; DD genotype: OR 4.50 , 95\% CI $1.96-$ $10.33 ; P<0.001$ ), recessive model (OR 4.10, 95\% CI 1.85-9.10; $P<0.001)$ and log-additive model (OR 1.69, 95\% CI 1.21-2.36; $P=0.002)$. The recessive model achieved the best scores for $\mathrm{AIC}$ and BIC.

The comparison between allergic patients and healthy controls did not show significant results for GSTO2*N142D. Conversely, for the GSTA1*-69C/T polymorphism, differences in genotype distribution were present. In particular, significant OR were obtained for the codominant model (CT genotype: OR 2.58, 95\% CI 1.40-4.77; TT genotype: OR 3.68, 95\% CI 1.38-9.81; $P=0.002$ ), dominant model (OR 2.76, 95\% CI 1.54-4.96; $P<0.001)$ and log-additive model (OR 2.12, 95\% CI 1.37-3.29; $P<0.001$ ). The dominant model achieved the best scores for $\mathrm{AIC}$ and BIC.

\section{DISCUSSION}

Asthma and respiratory allergies are complex diseases characterized by variable and subjective symptoms that are influenced by many genes and molecular mechanisms. The results of several studies have suggested the existence of different subphenotypes. ${ }^{9,27}$ However, more studies are needed to adequately define and characterize these respiratory diseases. In this scenario, environmental factors also play a fundamental role: asthma and allergies are, in fact, believed to be a result of the combined effects of genes, the environment and gene-environment interactions. $^{28,29}$ Asthma and allergies are mainly characterized by inflammatory conditions. Although respiratory allergies mainly distress upper airways with symptoms that predominantly affect the nose and eyes, asthma is primarly characterized by widespread but variable airflow limitation that is at least partially reversible with medication. ${ }^{28,29}$ Even though in ecent decades, several GWAS in asthma and allergy phenotypes have been published, their genetic component has not been completely solved. ${ }^{7}$ To detect new functional genes or to clarify the role of the genes that have been extensively studied, association studies are performed. In these studies, several candidate genes were directly or indirectly implicated, but it has been reported that the prevalence of these candidate genes varies considerably by ethnicity. ${ }^{20,26}$ The GST genes are important in the protection of cells from reactive oxygen species (ROS) and they can also affect the synthesis of proinflammatory eicosanoids via modulation of ROS levels. ${ }^{8}$

Our outcome suggests that GSTA1*-69C/T and GSTO2* $\mathrm{N} 142 \mathrm{D}$ are functional variants that affect the detoxification role of encoded enzymes, increasing the risk of respiratory disease development. Regarding asthma, our data confirm the association and strongly suggest a role in genetic predisposition for both variants investigated. Conversely, only GSTA1*-69C/T seems to be involved in respiratory allergy predisposition. The function of GSTA1 supports our results: GSTA1 is widely expressed in 
Table 3 Association of GSTA1 and GSTO2 variants with asthma and allergy

\begin{tabular}{|c|c|c|c|c|c|c|c|c|}
\hline & \multicolumn{4}{|c|}{ Asthmatic versus control } & \multicolumn{4}{|c|}{ Allergic versus control } \\
\hline & OR $(95 \% \mathrm{CI})$ & $P$-value & AIC & $\mathrm{BIC}$ & OR $(95 \% \mathrm{CI})$ & $P$-value & AIC & $\mathrm{BIC}$ \\
\hline Codominant & & $<0.001$ & 477 & 516 & & 0.002 & 307 & 344 \\
\hline$-69 \mathrm{C} /-69 \mathrm{~T}$ & $2.74(1.72-4.35)$ & & & & $2.58(1.40-4.77)$ & & & \\
\hline$-69 \mathrm{~T} /-69 \mathrm{~T}$ & $3.08(1.41-6.72)$ & & & & $3.68(1.38-9.81)$ & & & \\
\hline Log-additive & $2.10(1.48-2.97)$ & $<0.001$ & 478 & 513 & $2.12(1.37-3.29)$ & $<0.001$ & 306 & 339 \\
\hline \multicolumn{9}{|c|}{ GSTO2*N142D (rs156697) } \\
\hline Codominant & & $<0.001$ & 483 & 521 & & 0.800 & 319 & 355 \\
\hline N142/D142 & $1.20(0.76-1.89)$ & & & & $0.92(0.51-1.66)$ & & & \\
\hline D142/D142 & $4.50(1.96-10.33)$ & & & & $1.37(0.43-4.34)$ & & & \\
\hline Dominant & $1.51(0.98-2.33)$ & 0.062 & 492 & 527 & $0.97(0.55-1.72)$ & 0.920 & 316 & 349 \\
\hline
\end{tabular}

$P$-values and odds ratios (OR) adjusted for confounding variables (i.e. age, sex, body mass index, active and passive smoking and occupational risk) are reported.

Association analysis was performed considering different genetic models (i.e. codominant, dominant, recessive and log-additive).

Significance was set at $P=0.025$ (two-tailed after Bonferroni's adjustment).

several tissues (i.e. kidney, lung, small intestine, prostate, testis, adrenal gland, pancreas and trachea) and it is particularly expressed in the liver; this enzyme plays a fundamental role in the cellular detoxification of several phase I-activated derivatives of environmental pollutants or products of oxidative stress. ${ }^{30,31}$ The GSTA $1 *-69 \mathrm{C} / \mathrm{T}$ polymorphism is a single nucleotide polymorphism (SNP) localized in the proximal promoter and it is linked with other SNPs. It has been suggested that it may play a role in the development of asthma and allergies or the exacerbation of symptoms, because subjects with the $-69 \mathrm{~T}$ allele in the GSTA1 genotype exhibit lower GSTA1 expression. ${ }^{31}$ In this way, a deficiency in detoxifying air pollutants or allergens could play a role in the risk of atopic disease development. Regarding GSTO2, the encoding enzyme plays an important role in terms of response to oxidative stress through its dehydroascorbate reductase activity. ${ }^{32}$ This function is important for the oxidant-antioxidant imbalance present in lung tissue. ${ }^{33}$ Therefore, a decrease in anti-oxidant capacity due to reduced GSTO2 activity may trigger increased oxidative stress that could lead to a pulmonary diminution of its function. Wilk et al. ${ }^{24}$ in their GWAS on lung function have identified a number of novel gene regions associated with pulmonary function, in which the GSTO2*N142D polymorphism is included. Furthermore, this variant has shown significant association with forced expiratory volume in the first second $\left(\mathrm{FEV}_{1}\right)$ and forced vital capacity (FVC) phenotypes. ${ }^{34}$ It has been demonstrated that GSTO2 may exhibit expression in bronchial epithelial cells. In fact the GSTO2*D142 variant showed a minor percentage of expression with respect to the wild-type. ${ }^{24}$ Association studies on genes involved in lung function measurements and asthma may help identify lung-specific mechanisms in asthma development. These findings could help explain why GSTO2 seems to be linked only to asthma and not to allergies. Our hypothesis is that GSTO2 may be not involved in general inflammatory mechanisms, such as eosinophilia, that play a role in atopic diseases.
In conclusion, our investigation confirms the role of GSTA1 and GSTO2 loci in asthma risk and permits us to hypothesize that GSTA1 is a common susceptibility locus for asthma and allergies, whereas GSTO2 is an asthma-specific locus. The results obtained in this association study allowed us to open new directions of inquiry into the study of GST genes in respiratory diseases. In particular, further studies on GSTA and GSTO genes may uncover important gene-gene and gene-environment interactions in the pathogenesis of asthma and allergies.

\section{ACKNOWLEDGEMENTS}

The authors thank the volunteer participants for their generous cooperation and the collaborators of the 'San Giovanni Calibita' Fatebenefratelli Hospital for their valuable contributions. This study was supported by RSA Grant 2009 from University of Rome 'Tor Vergata', by PRIN 2009-11 (Project no. 200975T9EW) from the Italian Ministry of Instruction, University and Research (MIUR) and by a grant from the Fatebenefratelli Association for Biomedical Research (AFaR).

\section{DISCLOSURE}

The authors declare no conflicts of interest.

\section{REFERENCES}

1. Ober C, Hoffjan S. Asthma genetics 2006: The long and winding road to gene discovery. Genes Immun. 2006; 7: 95-100.

2. Rackeman FM. A working classification of asthma. Am. J. Med. 1947; 3: 601-6.

3. Godard P, Bousquet J, Michel FB. Extrinsic and intrinsic asthma: Still a matter of debate? Clin. Asthma Rev. 1997; 1: 19-22.

4. Humbert M, Menz G, Ying S et al. The immunopathology of extrinsic (atopic) and intrinsic (non-atopic) asthma: More similarities than differences. Immunol. Today 1999; 20: 528-33. 
5. Nieves A, Magnan A, Boniface $\mathrm{S}$ et al. Phenotypes of asthma revisited upon the presence of atopy. Respir. Med. 2005; 99: 347-54.

6. EFA. EFA Book on Respiratory Allergy in Europe: Relieve the Burden. 2011. Available from: http://www.efanet.org/category/publications/ (accessed 2 April 2013).

7. Binia A, Kabesch M. Respiratory medicine: Genetic base for allergy and asthma. Swiss Med. Wkly 2012; 142: w13612.

8. Gorska K, Krenke R, Domagala-Kulawik J et al. Comparison of cellular and biochemical markers of airway inflammation in patients with mild-to-moderate asthma and chronic obstructive pulmonary disease: An induced sputum and bronchoalveolar lavage fluid study. J. Physiol. Pharmacol. 2008; 59: 271-84.

9. Moffatt MF, Gut IG, Demenais F et al. A large-scale, consortiumbased genomewide association study of asthma. N. Engl. J. Med. 2010; 363: 1211-21.

10. Wjst M, Sargurupremraj M, Arnold M. Genome-wide association studies in asthma: What they really told us about pathogenesis. Curr. Opin. Allergy Clin. Immunol. 2013; 13: 112-18.

11. Stranger BE, Stahl EA, Raj T. Progress and promise of genomewide association studies for human complex trait genetics. Genetics 2011; 187: $367-83$.

12. Polimanti R, Piacentini S, Fuciarelli M. HapMap-based study of human soluble glutathione S-transferase enzymes. The role of natural selection in shaping the single nucleotide polymorphism diversity of xenobiotic-metabolizing genes. Pharmacogenet. Genomics 2011; 21: $665-72$

13. Piacentini S, Polimanti R, Squitti R et al. GSTO1*E 155 del polymorphism associated with increased risk for late-onset Alzheimer's disease: Association hypothesis for an uncommon genetic variant. Neurosci. Lett. 2012; 506: 203-7.

14. Piacentini S, Monaci PM, Polimanti R, Manfellotto D, Fuciarelli M. GSTO2*N142D gene polymorphism associated with hypothyroidism in Italian patients. Mol. Biol. Rep. 2013; 40: 1967-71.

15. Polimanti R, Piacentini S, Lazzarin N, Re MA, Manfellotto D, Fuciarelli M. Glutathione S-transferase variants as risk factor for essential hypertension in Italian patients. Mol. Cell. Biochem. 2011; 357: 227-33.

16. Polimanti R, Piacentini S, Lazzarin $\mathrm{N}$ et al. Glutathione S-transferase genes and the risk of recurrent miscarriage in Italian women. Fertil. Steril. 2012; 98: 396-400.

17. Gilliland FD, Li YF, Saxon A, Diaz-Sanchez D. Effect of glutathione-S-transferase M1 and P1 genotypes on xenobiotic enhancement of allergic responses: Randomised, placebo-controlled crossover study. Lancet 2004; 363: 119-25.

18. Piacentini S, Polimanti R, Moscatelli B, Re MA, Manfellotto D, Fuciarelli M. Lack of association between GSTM1, GSTP1, and GSTT1 gene polymorphisms and asthma in adult patients from Rome, central Italy. J. Investig. Allergol. Clin. Immunol. 2012; 22: $252-6$

19. Karam RA, Pasha HF, El-Shal AS, Rahman HM, Gad DM. Impact of glutathione-S-transferase gene polymorphisms on enzyme activity, lung function and bronchial asthma susceptibility in Egyptian children. Gene 2012; 497: 314-19.

20. Piacentini S, Polimanti R, Simonelli I et al. Glutathione S-transferase polymorphisms, asthma susceptibility and confounding variables: A meta-analysis. Mol. Biol. Rep. 2013; 40: 3299-313.
21. Minelli C, Granell R, Newson R et al. Glutathione S-transferase genes and asthma phenotypes. A Human Genome Epidemiology (HuGE) systematic review and meta-analysis including unpublished data. Int. J. Epidemiol. 2010; 39: 539-62.

22. Piacentini S, Polimanti R, Moscatelli B et al. Glutathione S-transferase gene polymorphisms and air pollution as interactive risk factors for asthma in a multicenter Italian field study: A preliminary study. Ann. Hum. Biol. 2010; 37: 427-39.

23. Piacentini S, Verrotti A, Polimanti R et al. Functional polymorphisms of GSTA1 and GSTO2 genes associated with asthma in Italian children. Clin. Chem. Lab. Med. 2012; 50: 311-15.

24. Wilk JB, Walter RE, Laramie JM, Gottlieb DJ, O'Connor GT. Framingham Heart Study genome-wide association: Results for pulmonary function measures. BMC Med. Genet. 2007; 8: S8.

25. Sohn SW, Jung JW, Lee SY et al. Expression pattern of GSTP1 and GSTA1 in the pathogenesis of asthma. Exp. Lung Res. 2013; 39 173-81.

26. Polimanti R, Carboni C, Baesso I et al. Genetic variability of glutathione S-transferase enzymes in human populations: Functional interethnic differences in detoxification systems. Gene 2013; 512: 102-7.

27. Kauffmann F, Demenais F. Gene-environment interactions in asthma and allergic diseases. Challenges and perspectives. J. Allergy Clin. Immunol. 2012; 130: 1229-40.

28. Bousquet J, Khaltaev N, Cruz AA. Allergic Rhinitis and its Impact on Asthma (ARIA) 2008 update. Allergy 2008; 63: 8-160.

29. Global Initiative for Asthma (GINA). Global Strategy for Asthma Management and Prevention. 2011. Available from: http://www. ginasthma.org/ (accessed 25 March 2012).

30. Coles BF, Kadlubar FF. Human alpha class glutathione S-transferases: Genetic polymorphism, expression, and susceptibility to disease. Methods Enzymol. 2005; 401: 9-42.

31. Morel F, Rauch C, Coles B, Le Ferrec E, Guillouzo A. The human glutathione transferase alpha locus: Genomic organization of the gene cluster and functional characterization of the genetic polymorphism in the hGSTA1 promoter. Pharmacogenetics 2002; 12: 277-86.

32. Schmuck EM, Board PG, Whitbread AK et al. Characterization of the monomethylarsonate reductase and dehydroascorbate reductase activities of Omega class glutathione transferase variants: Implications for arsenic metabolism and the age-at-onset of Alzheimer's and Parkinson's diseases. Pharmacogenet. Genomics 2005; 15: 493-501.

33. Mukherjee B, Salavaggione OE, Pelleymounter LL et al. Glutathione S-transferase omega 1 and omega 2 pharmacogenomics. Drug Metab. Dispos. 2006; 34: 1237-46.

34. Yanbaeva DG, Wouters EF, Dentener MA, Spruit MA, Reynaert NL. Association of glutathione-S-transferase omega haplotypes with susceptibility to chronic obstructive pulmonary disease. Free Radic. Res. 2009; 43: 738-43.

35. Polimanti R, Piacentini S, Moscatelli B, Pellicciotti L, Manfellotto D, Fuciarelli M. GSTA1, GSTO1, GSTO2 gene polymorphisms in Italian asthma patients. Clin. Exp. Pharmacol. Physiol. 2010; 37: $870-2$.

36. Budowle B, Moretti T, Smith J, Dizinno J. DNA Typing Protocols Molecular Biology and Forensic Analysis. Eaton Publishing, Natick, MA, 2000.

37. Menashe I, Rosenberg PS, Chen B. EPGA power calculator for case-control genetic association analyses. BMC Genet. 2008; 9: 36. 